Research Article

\title{
Study on Form-Finding of Cable-Membrane Structures Based on Particle Swarm Optimization Algorithm
}

\author{
Fangjin Sun $\mathbb{D}^{1,2}$ Donghan Zhu $\mathbb{D}^{1,}{ }^{3}$ Meirong Liang, ${ }^{3}$ and Daming Zhang ${ }^{4}$ \\ ${ }^{1}$ Guangxi Key Laboratory of New Energy and Building Energy Saving, Guilin 541004, China \\ ${ }^{2}$ College of Civil Engineering and Architecture, Guilin University of Technology, Guilin 541004, China \\ ${ }^{3}$ College of Civil Engineering, Liaoning Technical University, Fuxin 123000, China \\ ${ }^{4}$ College of Information Science and Engineering, Guilin University of Technology, Guilin 541006, China \\ Correspondence should be addressed to Fangjin Sun; e_dm@163.com
}

Received 18 December 2019; Revised 10 April 2020; Accepted 15 April 2020; Published 30 April 2020

Academic Editor: José Domingo Álvarez

Copyright (๑) 2020 Fangjin Sun et al. This is an open access article distributed under the Creative Commons Attribution License, which permits unrestricted use, distribution, and reproduction in any medium, provided the original work is properly cited.

\begin{abstract}
Form-finding is one of the key steps in the whole design process of cable-membrane structures. Traditional form-finding methods are usually complicated and have poor accuracy or stability. Thus, form-finding of cable-membrane structures based on particle swarm optimization (PSO) algorithm is proposed. The shape and loading characteristics of cable-membrane structures are optimized. The optimization objective is to minimize the maximum support reaction, and the initial prestress on the membrane surface is taken as the optimization variable. The main constraints are material strength limitation, structural stress, and displacement of the cable and membrane. A program is given based on the proposed PSO, and the optimization model and calculation process are implemented based on MATLAB and ANSYS platforms. Form-finding of three typical cable-membrane structures including rotating catenary surface, saddle surface, and rhombic hyperboloid is carried out. The results compare well with those from the force density method. The initial prestresses of the three membrane structures are obtained while the formfinding result is optimal, respectively. The proposed PSO shows a more accurate method in form-finding of cable-membrane structures in a simpler way.
\end{abstract}

\section{Introduction}

As a new type of modern public building structure, cablemembrane structures are widely used around the world. Cable-membrane structures are composed of high-strength flexible membrane materials and reinforcing members (such as steel columns and steel cables). In the engineering practice, through certain construction techniques and methods, different pretensioning stresses are generated inside the membrane material so that the membrane material has a certain solid shape [1].

Different from the traditional rigid building structures, the cable-membrane structure needs a certain initial prestress applied in order to form an initial shape form with sufficient rigidity and to support external loads [2]. Therefore, form-finding is the first key step in the whole design process of cable-membrane structures, followed by load analysis and clipping analysis. There are currently three commonly used form-finding methods of cable-membrane structures: force density method, dynamic relaxation method, and nonlinear finite element method [3], among which the force density method is widely used because of its simplicity compared with the other two methods. However, in the force density method, the appropriate value of its "force density" needs to be calculated and summarized through many trials before it can be determined, which makes the form-finding a complicated, less accurate, and a time-consuming process.

Due to the poor accuracy and complicated steps of the force density method, an alternative method is badly needed. In recent years, intelligent optimization methods are widely used in engineering applications, which paves a new way for form-finding of cable-membrane structures. Particle swarm optimization (PSO) algorithm [4-6], one of the intelligent 
optimization methods, has the merits of simpler principle, fewer parameters, and easier implementation. Thus, formfinding of cable-membrane structures based on PSO is proposed.

In this paper, particle swarm optimization (PSO)is employed to propose a form-finding optimization model for cable-membrane structures. The shape and loading characteristics of cable-membrane structures are optimized. The optimization objective is to minimize the maximum support reaction, and the initial prestress on the membrane surface is taken as the optimization variable. The main constraints are material strength limitation, structural stress, and displacement of the cable and membrane. A program is given based on the proposed PSO, and the optimization model and calculation process are implemented based on MATLAB and ANSYS platforms. Form-finding of three typical cablemembrane structures including rotating catenary surface, saddle surface, and rhombic hyperboloid is carried out.

\section{Theories for Form-Finding of Cable- Membrane Structures Based on Particle Swarm Optimization}

2.1. Classical Particle Swarm Optimization Algorithms. Particle swarm optimization (PSO) is an optimization algorithm proposed by Kennedy and Eberhart in 1995 [7]. In the particle swarm optimization (PSO) algorithm, the potential solutions to the considered optimization problem are called particles and fly through the solution domain. Particle swarm optimization is a stochastic optimization technique derived from swarm intelligence, compared with the genetic algorithm.

Suppose the search space of the problem to be solved is $D$ dimension and the total number of particles is $n$, then the position of the $i$ th particle can be expressed as $X_{i}=\left(x_{i 1}, x_{i 2}, \ldots, x_{i D}\right)$, where $D$ is the $D$-dimensional space. The best, most suitable, and advantageous position of the $i$ th particle in the history of "flight" (that is, the solution optimal corresponding to the position) is expressed as $P_{i}=\left(p_{i 1}, p_{i 2}, \ldots, p_{i D}\right)$, where it is assumed that $P_{g}$ is the optimal position found in the search for "flight" for all particles of $P_{i}(i=1, \ldots, n)$, that is, the optimal solution. The position change rate of the $i$ th particle (that is, the speed at which the $i$ th individual bird flies in search of food) is vector $V_{i}=\left(v_{i 1}, v_{i 2}, \ldots, v_{i D}\right)$. Through the analysis and summary, the position of each particle (that is, where each individual bird is in the food-seeking space) is changed according to the following formula ("flight"):

$$
\begin{gathered}
v_{i d}(t+1)=w \times v_{i d}(t)+c_{1} \times \operatorname{rand}() \times\left[p_{i d}(t)-x_{i d}(t)\right] \\
+c_{2} \times \operatorname{rand}() \times\left[p_{g d}(t)-x_{i d}(t)\right], \\
x_{i d}(t+1)=x_{i d}(t)+v_{i d}(t+1), 1 \leq i \leq n, 1 \leq d \leq D,
\end{gathered}
$$

where $c_{1}$ and $c_{2}$ are the normal numbers, called an acceleration factor, also known as a learning factor. On the one hand, it is to learn, develop themselves, and improve the ability to find food, that is, to search for the optimal solution and, on the other hand, is to learn from other bird groups social experience, to understand the trend of social development and development trends, correct their thoughts in a timely manner, and avoid falling into the bottleneck of local development, so as to better search for the best solution. $\operatorname{Rand}()$ is the random number between $[0,1]$ because the behavior of birds is random and unconstrained, providing infinite possibilities for finding the optimal result. $w$ is called the inertia factor. The larger $w$ is suitable for largescale exploration of the solution space, with fast search speed and improved search efficiency, while the smaller $w$ is suitable for small-scale exploitation, with high search accuracy to avoid missing the optimal solution so that the search results do not meet the requirements. The range of position variation of dimension $d$ is $\left[-X \mathrm{Max}_{d}, X \mathrm{Max}_{d}\right]$, which is the range of the minimum and maximum values of the solution space. The velocity range of each particle in the PSO algorithm is $\left[-V \mathrm{Max}_{d}, V \mathrm{Max}_{d}\right]$, which is the range of the minimum value and the maximum value of the position change of each bird in the bird flock; if the position and velocity of the particle exceed the boundary range in the iterative process, then the boundary value is taken. That is to say, if an individual bird flies out of the space where the food is located in the process of looking for food, or flies too fast and leaves the group, then the individual bird is pulled back to the space where the food is located and its flying speed is limited to the group. The entire algorithm calculation process is to first initialize the particle swarm and randomly generate the initial position and velocity of the particle swarm. Then, according to equations (1) and (2), iteration is carried out to update the position and speed of particle swarm until a satisfactory solution is found, that is, the birds find the food, the search process is completed, and the algorithm calculation process is ended.

The basic algorithm steps of PSO [7, 8] are as follows:

(1) Initialize the particle swarm according to the formula, randomly set the initial position $X_{i}$ and initial velocity $V_{i}$ of each particle, and provide the particle search algorithm with the possibility to search for the best results.

(2) Calculate the fitness value of each particle based on the basic principle of the algorithm.

(3) On this basis, update the best position $P_{i}$ of individual particles. The update criterion is for each particle, comparing its fitness value with the fitness value of the best position $P_{i}$ it has experienced and selecting the best one as the current fitness value.

(4) On this basis, update the best position $P_{c}$ of individual particles. The update criterion is for each particle, comparing the fitness value of each particle with the fitness value of the best position $P_{i}$ experienced by the group and selecting the best one as the current fitness value.

(5) Adjust the velocity $V_{i}$ and position $X_{i}$ of the particle according to equations (1) and (2).

(6) Finally, if the end conditions are met, the search process will be terminated and the search process will 
be terminated; if the end condition is not met, go to step (2) and continue the algorithm until the condition is met. Outputting the search results, the whole algorithm to solve the problem process is completed.

The basic algorithm flow chart of PSO is shown in Figure 1.

\subsection{Nonlinear Finite Element Theory of Cable-Membrane} Structures. The structural analysis of the cable-membrane structure in this paper uses the support lifting method in the nonlinear finite element method. The problem of forming the membrane structure is a static process, and the effect of external load is generally not considered. Therefore, the material mechanical properties (constitutive relation) of the cable and membrane are not considered here; only the size and distribution of the pretension related to the equilibrium state of the cable and membrane structure and the position of the supporting point are considered. Therefore, the nonlinear finite element analysis theory of the lower membrane structure is briefly introduced here.

\subsubsection{Establishing the Nonlinear Finite Element Equations of} Cable and Membrane Elements [9, 10]. The membrane unit here is a triangular membrane unit; firstly, the geometric functions of the cable and membrane elements and the geometric equations of the cable and membrane elements are determined in the local coordinate system; on this basis, the element balance equation of the cable and membrane structure in the local coordinate system is established, the coordinate transformation matrix is used to transform the local coordinate system into the global coordinate system, and the equilibrium equation of the structure under the global coordinate system is obtained.

Here, the displacement vector of the element in the global coordinate system, the equivalent load vector of the node, and the linear and nonlinear stiffness matrix are, respectively, represented by $\Delta U^{e}, F^{e}, K_{L}^{e}$, and $K_{N L}^{e}$. The load vector outside the element is expressed as $R^{e}$. The balance equation of the membrane element in the global coordinate system can be expressed as

$$
\left(K_{L}^{e}+K_{N L}^{e}\right) \Delta U^{e}=R^{e}-F^{e} .
$$

The equilibrium equation of the cable element in the global coordinate system is similar to that of the membrane element.

\subsubsection{Establishing the Structural Balance Equation of the} Whole Cable-Membrane Structure. Suppose that the node displacement vector, node equivalent load vector, linear and nonlinear stiffness matrix, and external load vector ( $n$ is the total number of elements) of the cable-membrane structure are represented by $\Delta U, F, K_{L}, K_{N L}$, and $R$, respectively, then for all elements (including the membrane element and cable element),

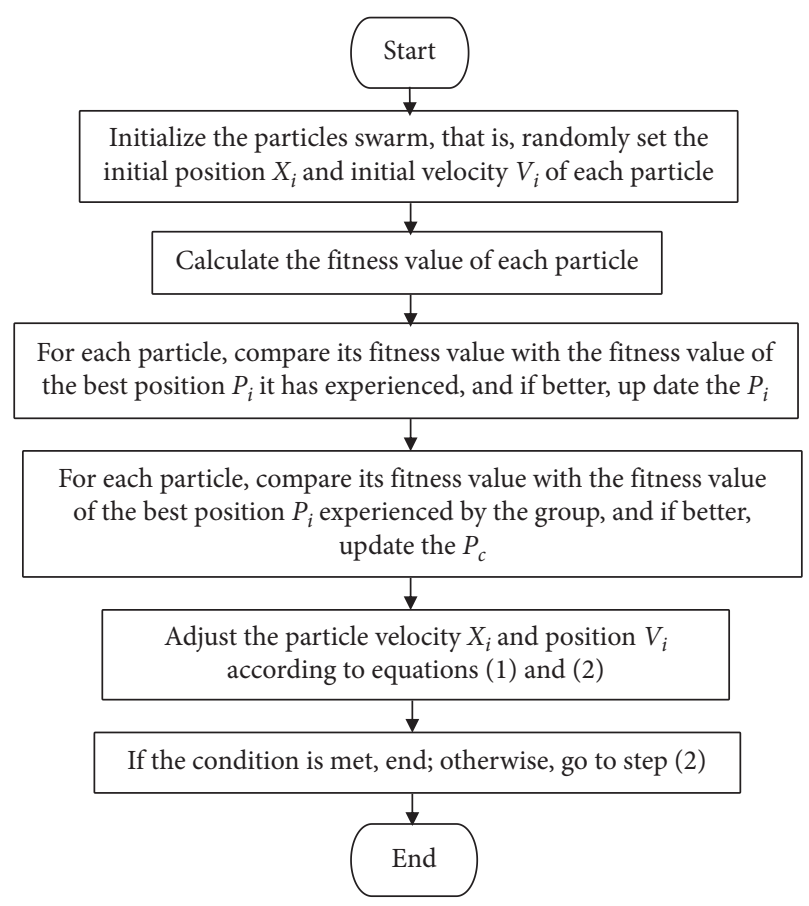

Figure 1: Classical PSO flow chart.

$$
\begin{gathered}
\Delta U=\sum_{i=1}^{n} \Delta U_{i}^{e}, \\
F=\sum_{i=1}^{n} F_{i}^{e}, \\
K_{L}=\sum_{i=1}^{n} K_{L i}^{e}, \\
K_{N L}=\sum_{i=1}^{n} K_{N L i}^{e}, \\
R=\sum_{i=1}^{n} R_{i}^{e} .
\end{gathered}
$$

The balance equation of the membrane element and the cable element in the global coordinate system is assembled into the balance equation of the cable-membrane structure in the global coordinate system, and it can be expressed as

$$
\left(K_{L}+K_{N L}\right) \Delta U=R-F \text {. }
$$

\subsection{Form-Finding Optimization Model Based on Particle Swarm Optimization}

2.3.1. Optimization Model of Cable-Membrane Structure. Based on the consideration of the "optimal form" [11, 12] of the cable-membrane structure and the finite element analysis of the cable-membrane structure, a model for the cablemembrane structure shape optimization is established. In the optimization model, there is a need to establish the objective function according to the optimization objective, to analyze the characteristics of the structure and to 
determine the optimization variables, to consider various factors affecting the determination of membrane structure shape in order to determine the constraint conditions.

Objective function: the optimization objective is to minimize the maximum bearing reaction force after loading (here, because it is the form-finding stage of the membrane structure, the load is the initial prestress applied on the surface of the membrane structure and the prestress applied on the cable, and the external load is 0 .)

The formula is written as

$$
\begin{aligned}
& N_{\max }=\sqrt{\left(N_{x}^{2}+N_{y}^{2}+N_{z}^{2}\right)}, \\
& f(X)=\min \left(N_{\max }\right),
\end{aligned}
$$

where $f(X)$ is the objective function, $N_{x}$ is the bearing reaction in the $x$ direction, $N_{y}$ is the bearing reaction in the $y$ direction, and $N_{z}$ is the bearing reaction in the $z$ direction.

After the objective function is determined, optimization variables are selected and constraint conditions are determined [13-15]. The optional optimization variables include the control point coordinates of the cablemembrane structure, the initial pretension value of each cable segment in the structure, and the initial pretension value in the membrane. At the same time, the design of cable-membrane structure should also follow certain conditions, that is, the restriction based on the consideration of the safety and design variables of the cablemembrane structure, which is called "constraint condition." In view of the characteristics of the cable-membrane structure, we select the optimization variable as the initial prestress $F_{i}$ of the membrane surface applied to the cablemembrane structure, and the initial prestress of the cable is expressed by the initial prestress of the membrane surface, which can reduce the optimization variable and simplify the establishment of the model formula. Here, the main constraint conditions are material strength limitation of cable and membrane, and structural stress and displacement constraint. Based on the abovementioned analysis, the derivation formula is as follows:

(1) Initial pretension of the film $F_{i}$ [13]: according to the thickness $t$ of the film material taken, the set material safety factor $\alpha<1$, the maximum tensile force value $F_{i \max }$ that the film can withstand is determined by calculation, and the minimum tension value $F_{i \text { min }}$ that should be maintained in the film is determined according to the requirements of the code; then, the constraint limit value of the initial prestress of the film can be obtained:

$$
F_{i \min }<F_{i}<F_{i \max } .
$$

(2) The initial pretension value of the cable $F_{c j}[16]$ :

$$
F_{c j}=\sigma \cdot t \cdot L \cdot\left(\frac{r}{2}+\frac{1}{8 \cdot r}\right)
$$

where $L$ represents the span required to be determined by the building shape, $r$ represents the ratio of vector to span, $\sigma$ represents the prestress in the film, and $t$ represents the thickness of the film material. The cable initial pretension value is determined by calculation.

(3) Node displacement constraint: $d_{K}^{F} \leq d_{\max }^{F}$.

(4) Stress constraint of the membrane element and the cable element:

$$
\begin{gathered}
\sigma_{m \text { min }}^{F} \leq \sigma_{i}^{F} \leq \sigma_{\text {max }}^{F}, \\
\sigma_{c \text { min }}^{F} \leq \sigma_{j}^{F} \leq \sigma_{c \text { max }}^{F} .
\end{gathered}
$$

According to the objective function, optimization variables and constraints on the basis of the analysis of the membrane structure for the shape optimization model are established, and then, the cable-membrane structure is optimized for shape finding by using common intelligent optimization algorithm-particle swarm optimization (PSO) combined with the nonlinear finite element method and the software MATLAB and ANSYS software programming.

\subsubsection{Parameter Setting of PSO}

(1) Initialize the particle swarm [17]: first, initialize the population. The content of the initialization population includes setting the population size of the population and the position and velocity of each particle in the population.

(2) Constraints processing method: based on the basic principle of particle swarm optimization algorithm, the position of particle $i$ is obtained, and the update equation of particle position is set. In this way, the optimal position of the individual particle and the global optimal position of the particle are selected through search and calculation.

Optimal position of individual particles:

$$
P_{i}(t)= \begin{cases}X_{i}(t), & \text { if } F\left(X_{i}(t)\right)<F\left(P_{i}(t-1)\right), \\ P_{i}(t-1), & \text { if } F\left(X_{i}(t)\right) \geq F\left(P_{i}(t-1)\right),\end{cases}
$$

where $P_{i}(t)$ represents the optimal individual position of particle $i$ at time $t, X_{i}(t)$ represents the current position of particle $i$ at time $t, P_{i}(t-1)$ represents the optimal individual position of particle $i$ at time $t-1$, $F\left(X_{i}(t)\right)$ represents the adaptive value of particle $i$ at time $t$, and $F\left(P_{i}(t-1)\right)$ represents the adaptive value of particle $i$ at time $t-1$.

When the adaptive value at time $t$ is less than the adaptive value at the optimal position of the individual at the previous moment, the optimal position of the individual is updated to the current position; otherwise, it remains the optimal position of the individual at the previous moment. 
The global optimal position of the particle:

$$
\begin{aligned}
g & =\arg \min _{1 \leq i \leq N} F\left(P_{i}(t)\right), \\
G(t) & =P_{g}(t),
\end{aligned}
$$

where $g$ represents the particle number with the minimum adaptive value of the optimal position of each individual; $\min F\left(P_{i}(t)\right)$ represents the minimum adaptive value of the optimal position of each individual; arg represents the particle number corresponding to the extraction of the adaptive value; $G(t)$ represents the global optimal position at time $t$; and $P_{g}(t)$ represents the optimal position of the individual with the minimum adaptive value at time $t$.

Since this problem is a constrained optimization problem, the dynamic penalty function is used for the shape-finding optimization of the membrane structure, and the adaptive value of the particle is expressed as

$F\left(X_{i}(t)\right)=f\left(X_{i}(t)\right)+r^{t}\left(\sum_{k=1}^{n_{g}}\left[G_{k}\left(X_{i}(t)\right)^{2}\right]\right)$,

where $F\left(X_{i}(t)\right)$ represents the adaptive value of the $i$ th particle at time $t, f\left(X_{i}(t)\right)$ represents the objective function value of the $i$ th particle at time $t, r$ is the penalty factor, $r=2.0, G_{k}\left(X_{i}(t)\right)$ represents the $k$ th constraint violation of the $i$ th particle at time $t$, and $n_{g}$ represents the total number of constraints.

The constraint violation quantity is defined as follows:

$G_{k}\left(X_{i}(t)\right)=\max \left(g_{k}\left(X_{i}(t)\right), 0\right), \quad k=1,2, \ldots, n_{g}$.

(3) Particle transboundary processing: when the particle is updated in position, it may cross the boundary of geometric constraint or performance constraint, so it is necessary to transgress the boundary in order to maintain the population size. For geometric constraint processing, the flying back strategy is adopted to pull the out-of-bounds particles back to a random position within the feasible region:

$$
\left.\begin{array}{ll}
X_{i, j}(t)=X_{j}^{L}+r\left(X_{j}^{U}-X_{j}^{L}\right), & \text { if } X_{i, j}<X_{j}^{L} \\
X_{i, j}(t)=X_{j}^{U}-r\left(X_{j}^{U}-X_{j}^{L}\right), & \text { if } X_{i, j}>X_{j}^{U}
\end{array}\right\},
$$

where $r$ is the random number that obeys the distribution of $U(0,1)$ and $X_{j}^{U}$ and $X_{j}^{L}$ represent the upper and lower limits of the $j$-dimension, respectively.

\subsubsection{Particle Swarm Optimization Form-Finding Steps and Flow Chart}

(1) Optimization steps:

(i) Initialize the population: a set of individuals are randomly generated in the optimization interval to form the initial population, and each individual corresponds to a set of optimization variables. (ii) The nonlinear finite element method is used to conduct form-finding analysis for each individual in the population, and the objective function values of each individual are obtained.

(iii) For each individual in the population, that is, particles, particle swarm optimization algorithm is adopted to carry out the operation. Based on the comparison and selection, the optimal position of individual particles and the global optimal position are obtained.

(iv) Reach the iteration condition and output the optimal result.

(v) Input the data obtained from the optimal results into ANSYS to obtain the stress distribution diagram of the membrane surface, the support reaction force, etc.

(2) Optimize the flow chart, as shown in Figure 2.

\section{Form-Finding Analysis of Cable-Membrane Structures Based on Particle Swarm Optimization Algorithm}

Based on the optimization model of the cable-membrane structure based on particle swarm optimization algorithm established in the previous chapter, the optimization analysis of several typical cable-membrane structures is carried out, and the optimization results obtained from the optimization model are compared with the force density method to prove its feasibility, correctness, and effectiveness.

3.1. Rhombic Hyperboloid Cable-Membrane Structure. The structure shape of the rhombic hyperboloid cable membrane is square, as shown in Figure 3 [18]. Its diagonal distance is $10 \mathrm{~m}$, its height is $4 \mathrm{~m}$, and the material parameters of the structure are as follows: the initial pretension of the film surface is $\sigma=20 \mathrm{~N} / \mathrm{cm}$, the tensile strength is $E_{t}=2550 \mathrm{~N} / \mathrm{cm}$, the shear stiffness is $G_{t}=800 \mathrm{~N} / \mathrm{cm}$, and Poisson's ratio is $\gamma=0.3 .4$ corners of the structure are fixed, 4 edges are flexible side cables, and the initial pretension of side cables is $30 \mathrm{kN}, \mathrm{EA}=3 \times 10^{4} \mathrm{kN}$. The equation of the surface under the coordinates used in this paper is

$$
z=f \cdot\left[\frac{(x-5)^{2}}{a^{2}}-\frac{y^{2}}{b^{2}}\right]-2,
$$

where $f=2, a=5 \mathrm{~m}$, and $b=5 \mathrm{~m}$.

Particle swarm optimization (PSO) is used to optimize the membrane structure and the results are compared with those of the traditional force density method. Figure 4 shows the mesh division diagram for form-finding, Figure 5 shows the form-finding morphology diagram based on ANSYS, and Figure 6 shows the membrane surface stress diagram based on ANSYS. Table 1 shows the calculation results and analytical values determined by the particle swarm optimization algorithm for the structure shape; Table 2 shows the calculation results and analytical values determined by the force density method for the structure shape; and Table 3 shows the support reaction force obtained by using ANSYS 


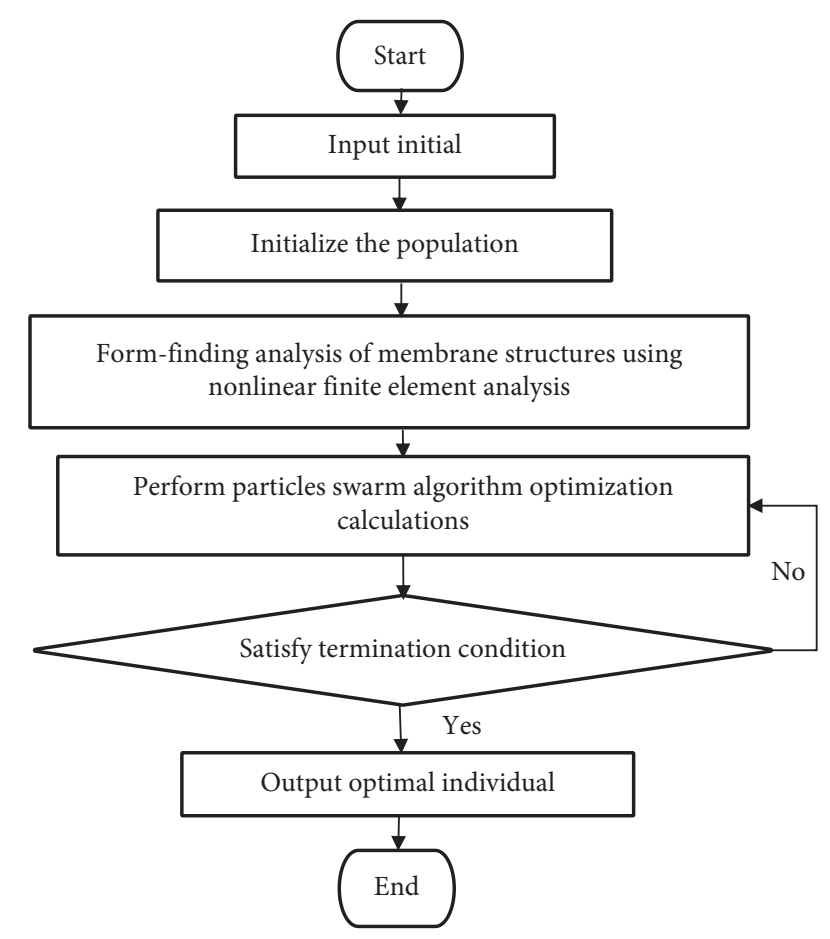

Figure 2: Optimization of the flow chart.

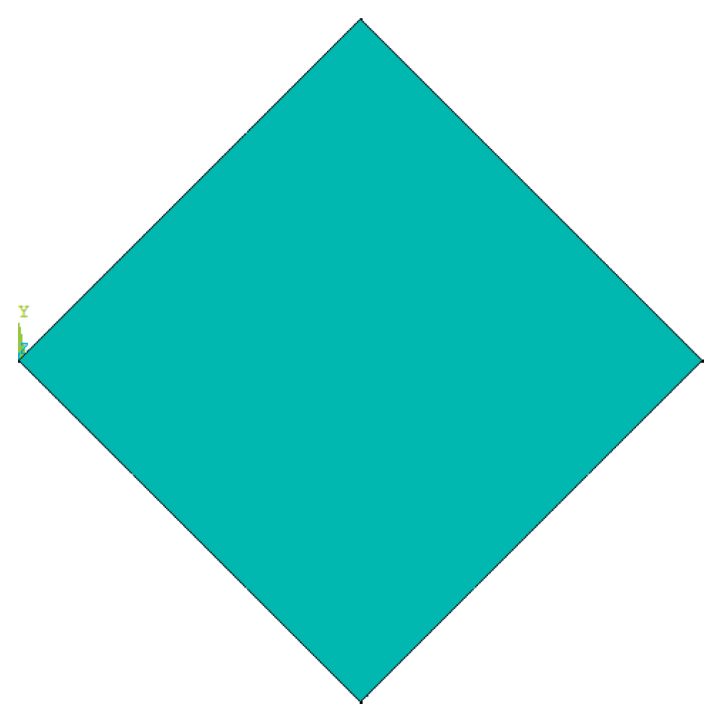

Figure 3: Planar projection of rhomboid hyperboloid.

software for the initial prestress of the membrane based on the particle swarm optimization algorithm.

\subsection{Form-Finding Optimization Analysis of Rotating Cate-} nary Cable-Membrane Structure. Select the catenary surface, a minimal surface with an analytical solution [19]. As shown in Figure 7, it is the projection plan of the structure of the rotating catenary mask, and its surface equation is as follows:

$$
z=h-a\left[\ln \left(\sqrt{x^{2}+y^{2}}+\sqrt{x^{2}+y^{2}-a^{2}}\right)-\ln a\right]
$$

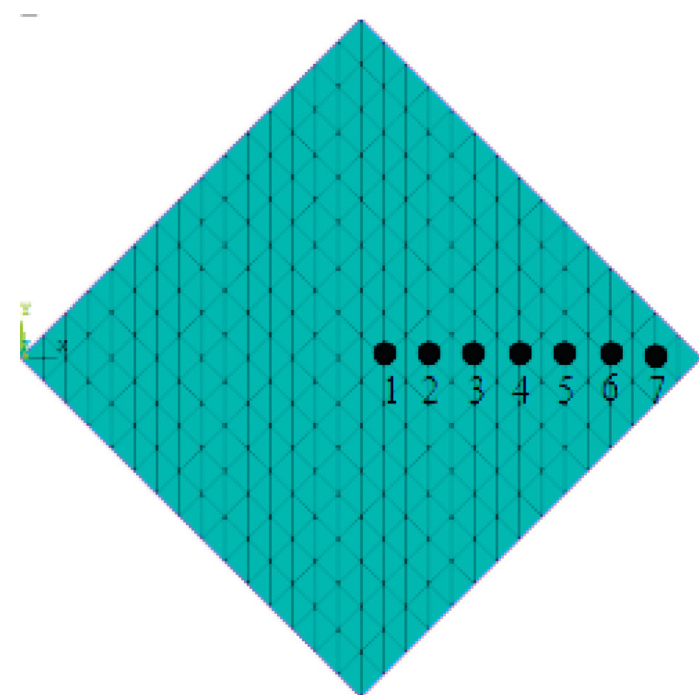

FIGURE 4: Meshing diagram of rhomboid hyperboloid.

where $h=22.9243 \mathrm{~m}, a=10 \mathrm{~m}$, and the outer ring is $50 \mathrm{~m}$.

The particle swarm optimization algorithm is used to optimize the form-finding of the cable-membrane structure, and the results are compared with the traditional force density method. Figure 8 shows the mesh division used for formfinding, Figure 9 shows the form-finding shape diagram obtained based on ANSYS software, and Figure 10 shows the stress distribution diagram of the catenary mask surface obtained based on ANSYS software. Table 4 shows the calculation results and analytical values determined by particle swarm optimization for the structure shape, Table 5 shows the calculation results and analytical values determined by the force density method for the structure shape, and Table 6 shows the support reaction force obtained by using ANSYS software for the initial prestress of the membrane based on the particle swarm optimization algorithm.

3.3. Form-Finding Optimization Analysis of Saddle Face Cable-Membrane Structure. The shape of the cable-membrane structure of the saddle is shown in Figure 11 [20]. The two long edges of the structure are circular curved fixed boundaries with a length of $31.4 \mathrm{~m}$, and the two short edges are direct fixed boundaries with a length of $20 \mathrm{~m}$. The results are obtained by using ANSYS.

Particle swarm optimization (PSO) is used to optimize the structure of the cable membrane. The optimized result is compared with that of traditional force density method. Figure 12 shows the mesh division used for saddle surface formfinding, Figure 13 shows the form-finding shape diagram based on ANSYS software, and Figure 14 shows the stress distribution diagram of the membrane surface based on ANSYS software. Table 7 shows the calculation results of the particle swarm optimization algorithm for the determination of the structure shape, Table 8 shows the calculation results of the force density method for the determination of the structure shape, and Table 9 shows the support reaction force obtained by using ANSYS software for the initial prestress of the membrane based on the particle swarm optimization algorithm. 


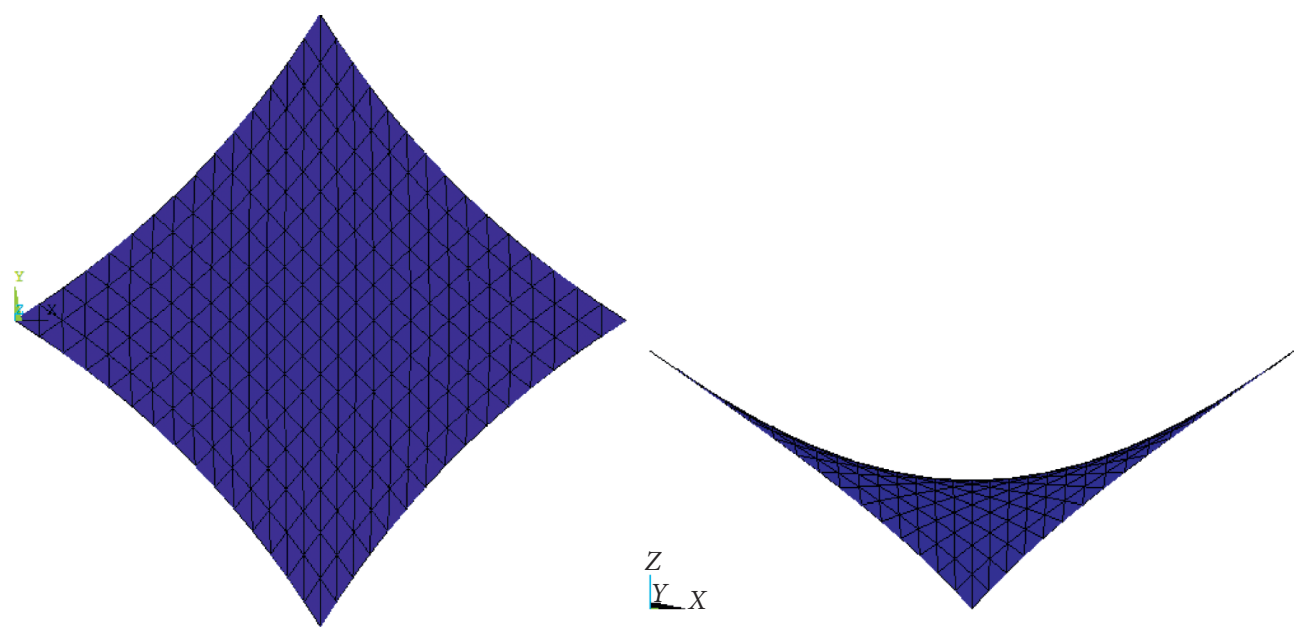

FIGURE 5: Form-finding of rhombic hyperboloid.

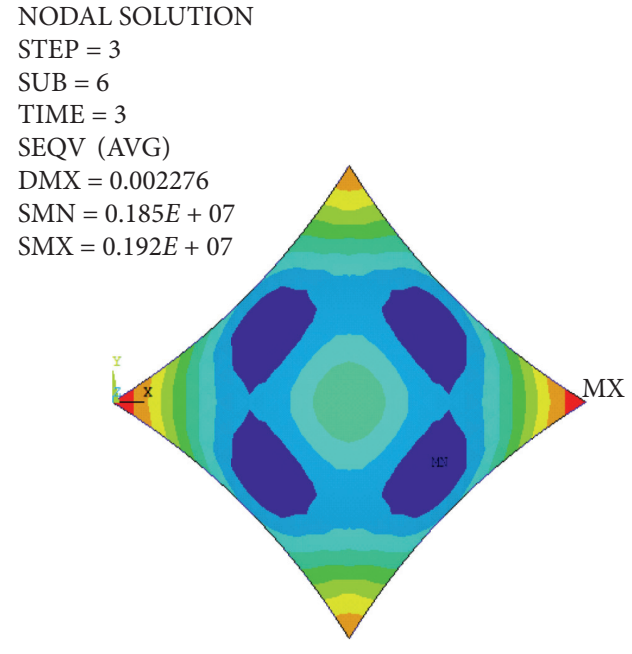

(a)

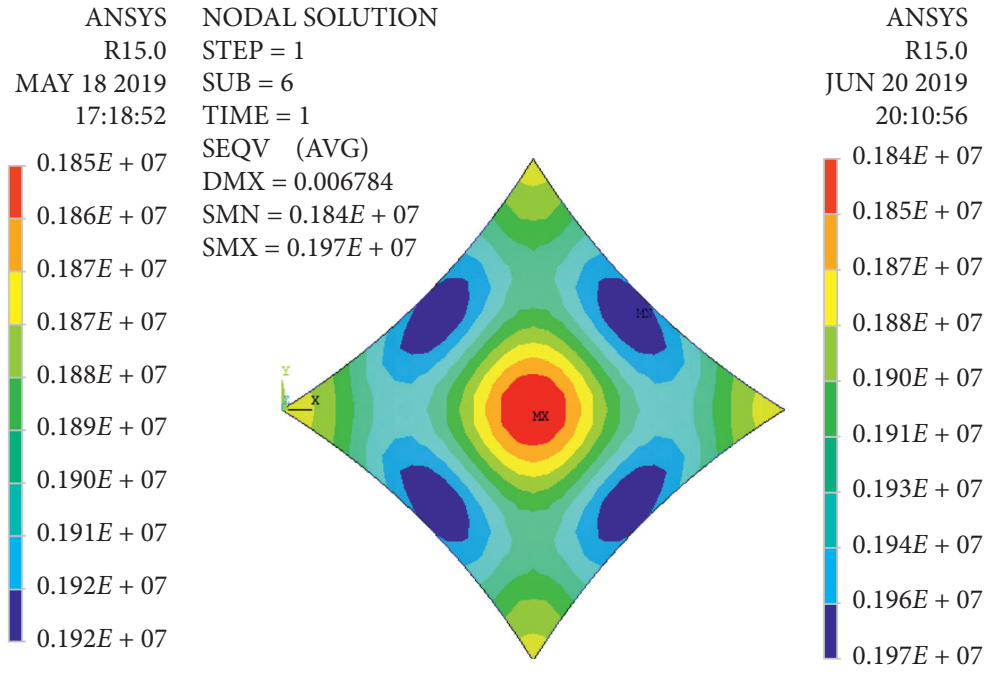

(b)

FIgURE 6: Stress distribution diagrams of the rhombic hyperbolic mask. (a) Particle swarm optimization algorithm form-finding model. (b) Force density method.

TABLE 1: Results and analytical values of particle swarm optimization for structural morphology determination.

\begin{tabular}{lccccc}
\hline Node & $X$ & $Y$ & $Z$ & $Z$ (analytical value) & Error (\%) \\
\hline 1 & 5.389 & $1.72 E-15$ & -1.992 & -1.988 & 0.21 \\
2 & 5.972 & $1.48 E-15$ & -1.934 & -1.924 & 0.50 \\
3 & 6.575 & $1.81 E-15$ & -1.814 & -1.802 & 0.69 \\
4 & 7.206 & $6.55 E-16$ & -1.624 & -1.611 & 0.82 \\
5 & 7.872 & $6.84 E-16$ & -1.351 & -1.340 & 0.80 \\
6 & 8.577 & $2.15 E-15$ & -0.988 & -0.976 & 1.17 \\
7 & 9.317 & $9.77 E-15$ & -0.534 & -0.509 & 4.67 \\
\multicolumn{5}{l}{ Maximum error } \\
\hline
\end{tabular}

TABLE 2: Calculation results and analytical values of force density method for determination of structural morphology.

\begin{tabular}{lccccc}
\hline Node & $X$ & $Y$ & $Z$ & $Z$ (analytical value) & Error (\%) \\
\hline 1 & 5.489 & $1.64 E-15$ & -1.992 & -1.981 & 0.56 \\
2 & 5.972 & $1.78 E-15$ & -1.932 & -1.924 & 0.39 \\
3 & 6.674 & $1.93 E-15$ & -1.808 & -1.776 & 1.78 \\
4 & 7.304 & $2.98 E-15$ & -1.613 & -1.575 & 2.34 \\
5 & 7.969 & $5.99 E-16$ & -1.339 & -1.295 & 3.30 \\
6 & 8.675 & $1.61 E-15$ & -0.978 & -0.920 & 5.98 \\
7 & 9.327 & $6.36 E-16$ & -0.529 & -0.502 & 5.07 \\
\multicolumn{5}{l}{ Maximum error } \\
\hline
\end{tabular}


TABLE 3: Reaction force of the rhombic-shaped hyperbolic form-finding support.

\begin{tabular}{lccc}
\hline Node & Reaction force in the $X$ direction $(\mathrm{kN})$ & Reaction force in the $Y$ direction $(\mathrm{kN})$ & Reaction force in the $Z$ direction $(\mathrm{kN})$ \\
\hline Left corner & 47.843 & $0.16 E-10$ & -33.444 \\
Upper corner & $0.12 E-07$ & -47.851 & 33.444 \\
Bottom corner & $0.19 E-09$ & 47.851 & 33.444 \\
Right corner & -47.843 & $0.37 E-07$ & -33.444 \\
\hline
\end{tabular}

Note. The node number is in the plane projection of the surface, starting from the central point of the structure (excluding the central point of the structure), and the node number is from the inner circle to the outer circle along the $x$-axis forward (excluding the corner point) [8].
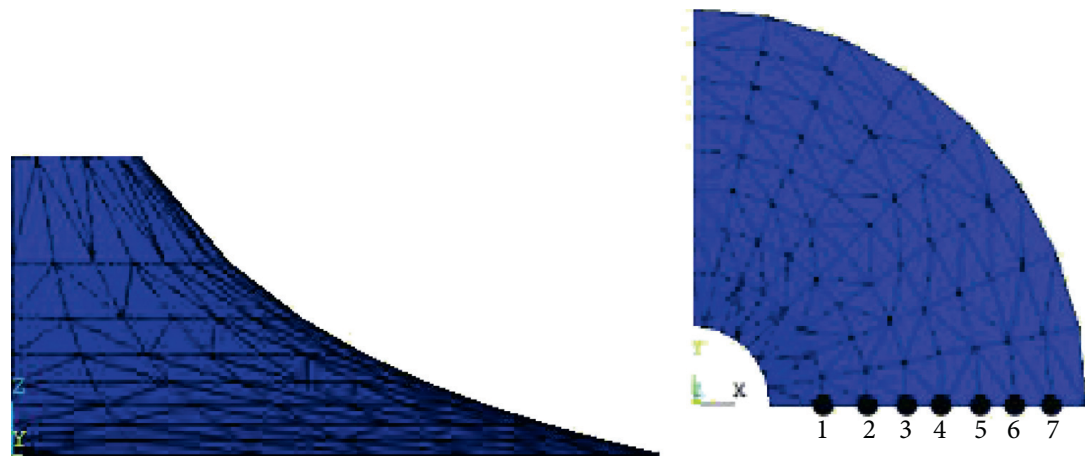

Figure 7: Projection plan of the rotating catenary.

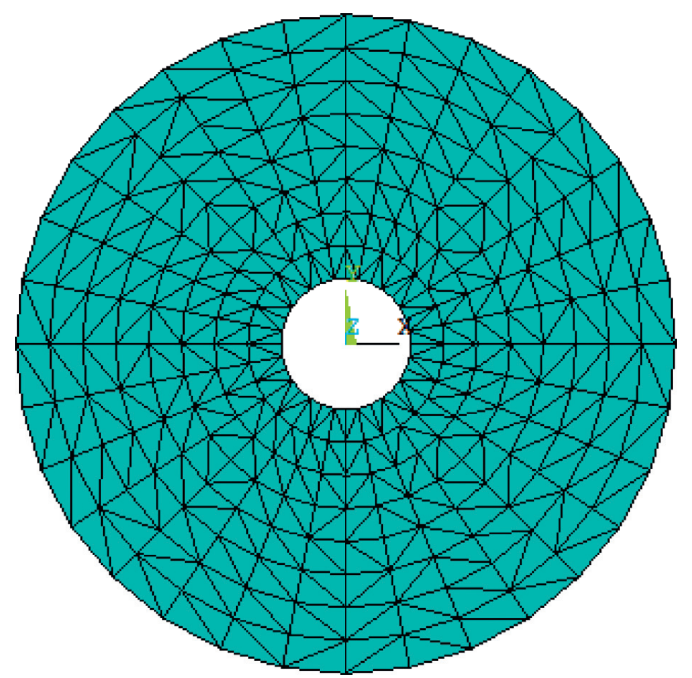

FIGURE 8: Grid division diagram of the rotating catenary surface.
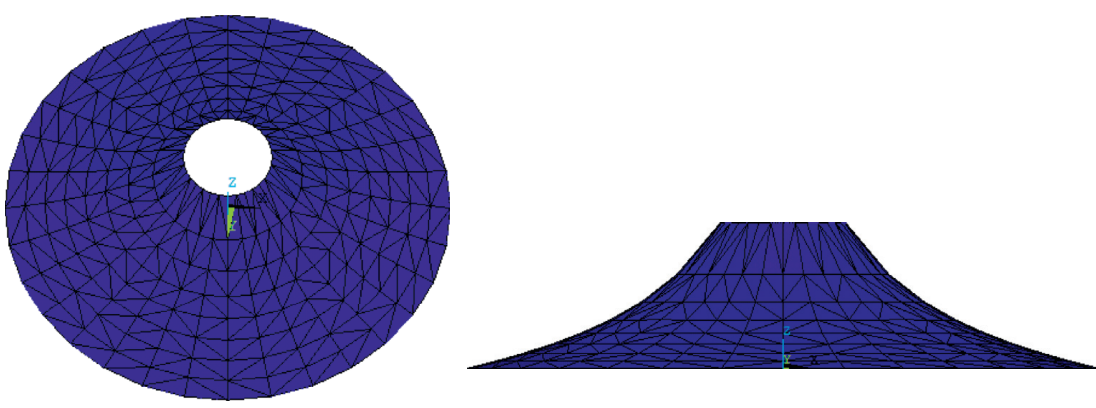

FIGURE 9: Form-finding pattern of rotary catenary. 


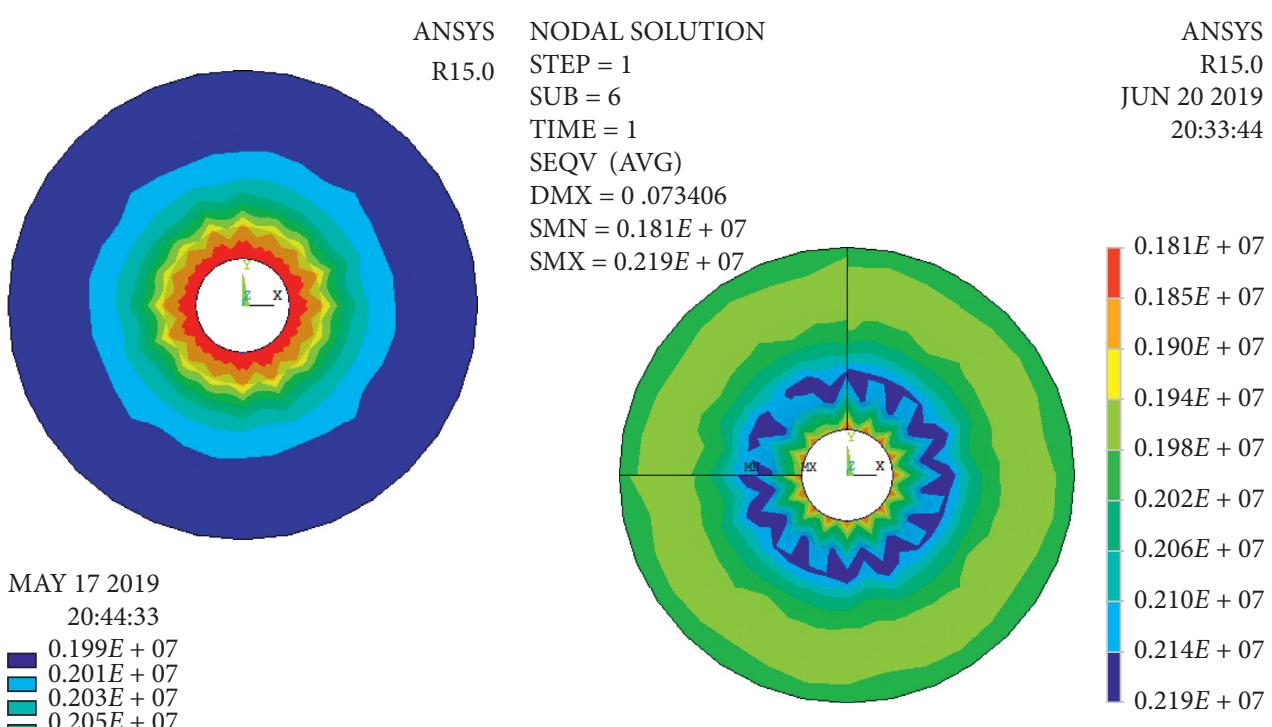

(a)

(b)

FIgURE 10: Stress distribution diagrams of the rotating catenary mask. (a) Particle swarm optimization algorithm form-finding model. (b) Force density method.

TABLE 4: Results and analytical values of particle swarm optimization for structural morphology determination.

\begin{tabular}{lccccc}
\hline Node & $X$ & $Y$ & $Z$ & $Z$ (analytical value) & Error (\%) \\
\hline 1 & 10.868 & 2.708 & 18.097 & 18.072 & 0.14 \\
2 & 13.628 & 3.447 & 14.630 & 14.196 & 2.97 \\
3 & 16.889 & 4.124 & 11.808 & 11.417 & 3.31 \\
4 & 20.65 & 5.262 & 9.323 & 9.029 & 3.15 \\
5 & 25.411 & 5.877 & 6.977 & 6.796 & 2.59 \\
6 & 31.172 & 8.474 & 4.670 & 4.516 & 3.30 \\
7 & 38.433 & 11.559 & 2.301 & 2.255 & 2.00 \\
\multicolumn{5}{l}{ Maximum error } \\
\multicolumn{5}{l}{}
\end{tabular}

TABLE 5: Calculation results and analytical values of the force density method for determination of structural morphology.

\begin{tabular}{lccccc}
\hline Node & $X$ & $Y$ & $Z$ & $Z$ (analytical value) & Error (\%) \\
\hline 1 & 37.433 & 10.559 & 2.866 & 2.58 & 9.98 \\
2 & 29.172 & 8.474 & 5.731 & 5.164 & 9.89 \\
3 & 22.411 & 7.377 & 8.597 & 7.892 & 8.20 \\
4 & 17.15 & 6.262 & 11.462 & 10.825 & 5.56 \\
5 & 14.389 & 5.124 & 14.328 & 13.059 & 8.86 \\
6 & 12.128 & 3.447 & 17.193 & 15.85 & 7.81 \\
7 & 10.868 & 2.708 & 20.059 & 18.072 & 9.91 \\
\multicolumn{5}{l}{ Maximum error } \\
\hline
\end{tabular}

\section{Results and Discussion}

4.1. Rhombic Hyperboloid Membrane Structure. Results analysis: the optimal result is obtained when the initial prestress applied is $20 \mathrm{~N} / \mathrm{cm}$ according to the optimization model of cable-membrane structure form-finding based on particle swarm optimization algorithm. It can be seen from Figure 5 that the surface of the form-finding morphological graph is relatively flat and its curvature is relatively small. According to Figure 6(a), it can be obtained that the maximum stress value on the membrane surface is $1.92 \mathrm{kN} /$ $\mathrm{m}$, and the minimum stress value on the membrane surface is $1.85 \mathrm{kN} / \mathrm{m}$, with a difference of $3.65 \%$ between the two sizes. Thus, it can be seen that the stress distribution on the membrane surface is relatively uniform. Comparing and contrasting the results of Tables 1 and 2 and the analytical solution, it can be seen that the maximum error between the nodal coordinate value and the theoretical solution value of rhomboid hyperboloid generated by particle swarm optimization algorithm is $4.67 \%$ and the maximum error is smaller than that obtained by the force density method. Therefore, the particle swarm algorithm to get the results of the calculation accuracy is higher than the traditional force density method. By comparing the calculated results in Tables 1 and 2 with the analytical solutions, it is found that the particle swarm optimization algorithm is feasible. By comparing the bearing reaction obtained in Table 3 with that obtained by professional software in the literature, there is little difference. Comparing with other bearing reaction obtained by applying initial prestress, this is the smallest, which can prove that this method is correct.

Conclusions: according to the above analysis results, when the initial prestress applied to the membrane is $20 \mathrm{~N} / \mathrm{cm}$, the form-finding result is optimal. It is feasible to use the particle swarm optimization model to optimize the formfinding of the rhomboid hyperboloid with high calculation 
TABLE 6: Reaction force of the rotating catenary surface form-finding support.

\begin{tabular}{lccc}
\hline Node & Reaction force in the $X$ direction $(\mathrm{kN})$ & Reaction force in the $Y$ direction $(\mathrm{kN})$ & Reaction force in the $Z$ direction $(\mathrm{kN})$ \\
\hline 1 & $-0.11131 E-01$ & 3.2134 & 4.3685 \\
2 & 0.0076 & 24.613 & -4.4950 \\
10 & 24.613 & -0.0076 & -4.4950 \\
15 & 13.475 & 20.480 & -4.5011 \\
18 & 3.2134 & $0.11131 E-01$ & 4.3685 \\
82 & -0.0076 & -24.613 & -4.4950 \\
90 & $0.11131 E-01$ & -3.2134 & 4.3685 \\
162 & -3.2134 & $-0.11131 E-01$ & 4.3685 \\
\hline
\end{tabular}

Note. The node number is in the plane projection of the surface, starting from the central point of the structure (excluding the central point of the structure) and the node number is from the inner circle to the outer circle along the $x$-axis forward (excluding the corner point).

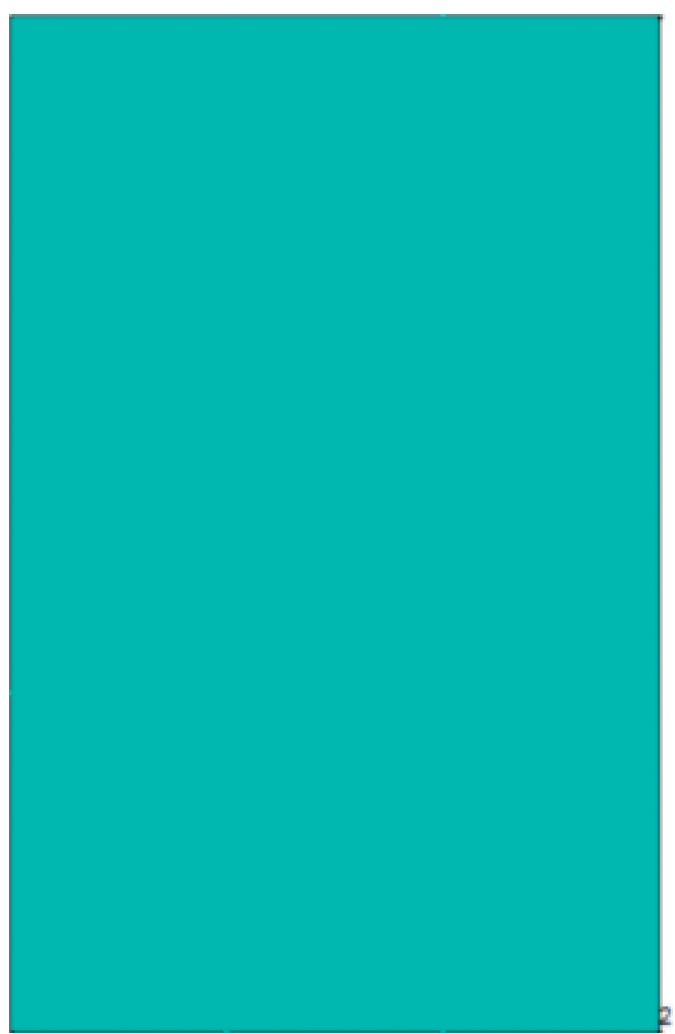

FIGURE 11: Projection plan of the saddle surface.

accuracy, and it is easy to operate, easy to implement, and fast to calculate.

4.2. Rotating Catenary Membrane Structure. Results analysis: according to the optimization model of cable-membrane structure form-finding based on particle swarm optimization algorithm, the form-finding result of the rotating catenary surface is optimal when the initial prestress applied is $20 \mathrm{~N} / \mathrm{cm}$. It can be seen from Figure 9 that the surface of the form-finding morphological graph is relatively flat and smooth, and its curvature is relatively small. According to Figure 10(a), it can be obtained that the maximum stress value of the membrane surface is $2.14 \mathrm{kN} / \mathrm{m}$, and the minimum stress value of the membrane surface is $1.99 \mathrm{kN} / \mathrm{m}$. The error difference between the two sizes is $7.01 \%$.

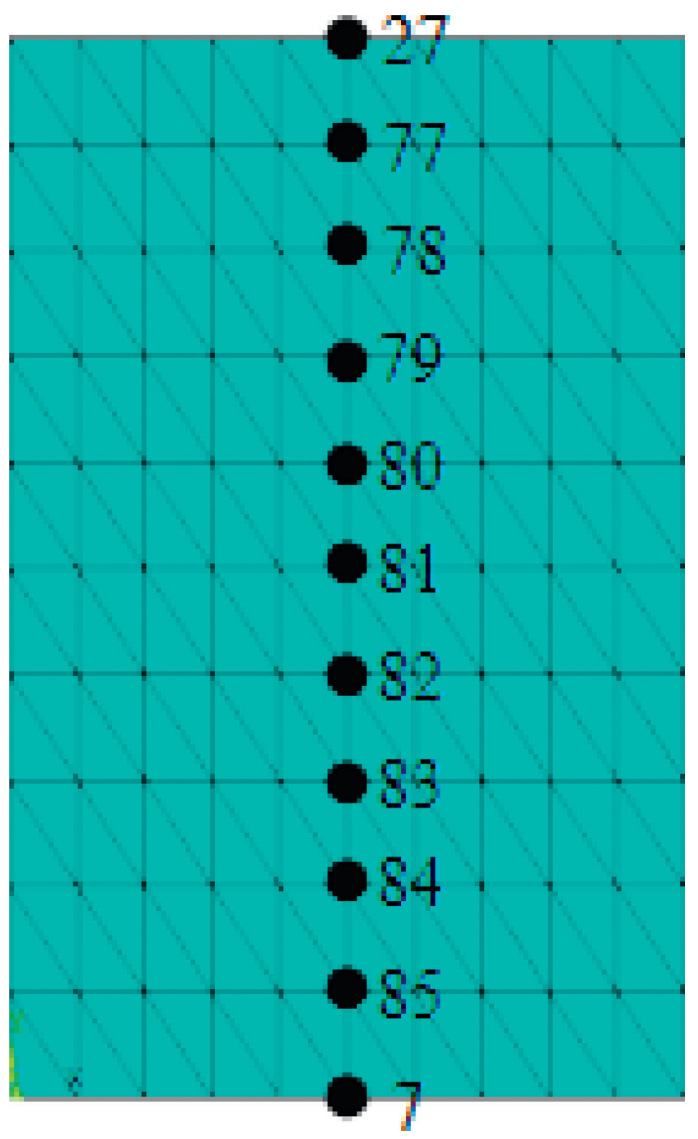

Figure 12: Saddle mesh division diagram.

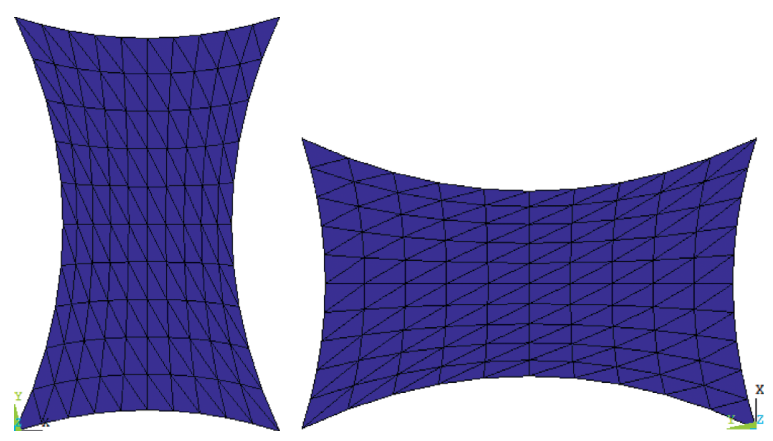

FIGURE 13: Saddle surface form-finding pattern. 


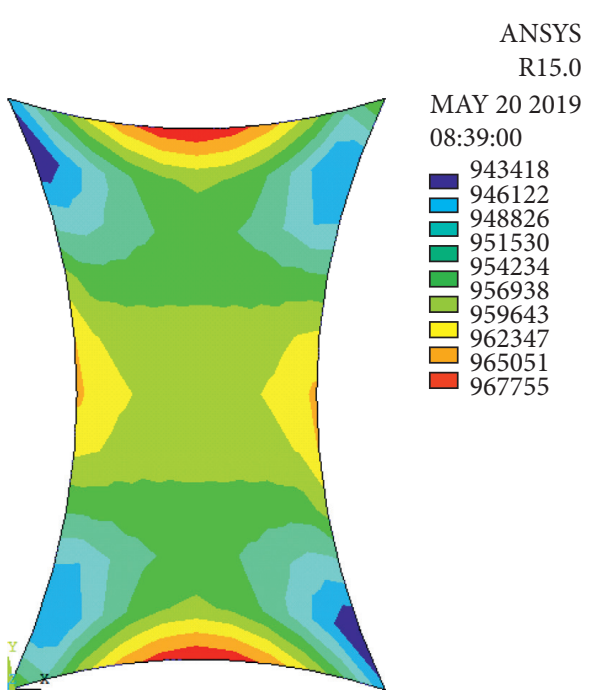

(a)

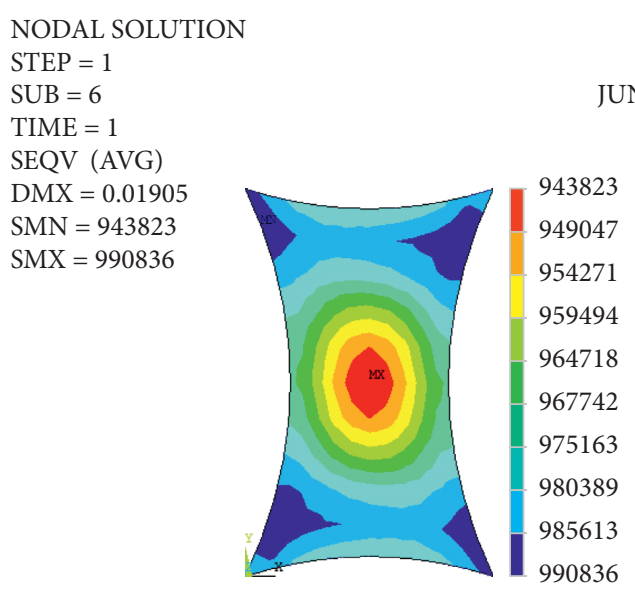

(b)

Figure 14: Saddle mask face stress distribution. (a) Particle swarm optimization algorithm form-finding model. (b) Force density method.

TABLE 7: Calculation results of particle swarm optimization for structural morphology determination.

\begin{tabular}{lccc}
\hline Node & $X$ & $Y$ & $Z$ \\
\hline 7 & 10.001659 & 1.608521 & -2.001515 \\
85 & 9.999437 & 4.317881 & -1.999383 \\
84 & 9.999712 & 7.134952 & -1.998242 \\
83 & 10.000081 & 9.986165 & -1.997328 \\
82 & 10.000153 & 12.843590 & -1.996686 \\
81 & 10 & 15.7 & -1.996453 \\
80 & 9.999847 & 18.556410 & -1.996686 \\
79 & 9.999919 & 21.413835 & -1.997328 \\
78 & 10.000288 & 24.265047 & -1.998242 \\
77 & 10.000563 & 27.082119 & -1.999383 \\
27 & 9.998341 & 29.791479 & -2.001515 \\
\hline
\end{tabular}

TABLE 8: Calculation results of the force density method for determination of structure morphology.

\begin{tabular}{lccc}
\hline Node & $X$ & $Y$ & $Z$ \\
\hline 7 & 10.001716 & 1.607515 & -2.000918 \\
85 & 9.999519 & 4.318502 & -1.999654 \\
84 & 9.999747 & 7.136213 & -1.999196 \\
83 & 10.000076 & 9.987353 & -1.998890 \\
82 & 10.000142 & 12.844280 & -1.998663 \\
81 & 10 & 15.7 & -1.998573 \\
80 & 9.999858 & 18.555720 & -1.998663 \\
79 & 9.999924 & 21.412647 & -1.998890 \\
78 & 10.000253 & 24.263787 & -1.999196 \\
77 & 10.000481 & 27.081498 & -1.999654 \\
27 & 9.998284 & 29.792485 & -2.000918 \\
\hline
\end{tabular}

Comparing the calculation results and the analytical solution (Tables 4 and 5), it can be seen that the maximum error between the calculated results of the rotating catenary generated by particle swarm optimization (PSO) and the theoretical analytical solution is $3.31 \%$ and the maximum error of the calculated result obtained by the specific force density method is $9.98 \%$ smaller; therefore, the particle swarm algorithm to get the results of the calculation accuracy is higher than the traditional force density method. According to the comparison between the calculated results in Tables 4 and 5 and the analytical solution, the particle swarm optimization algorithm is feasible. Comparing the bearing reaction obtained in Table 6 with that obtained by applying initial prestress, this is the smallest, which proves that this method is correct and the form-finding result is optimal.

Conclusions: according to the analysis of the abovementioned results, it is shown that the form-finding optimization model of the cable-membrane structure based on the particle swarm optimization algorithm is feasible for form-finding optimization of the rotating catenary surface, and it is easy to operate, easy to implement, and fast to calculate, and when the initial prestress applied is $20 \mathrm{~N} / \mathrm{cm}$, the shape-finding result is optimal.

4.3. Saddle Membrane Structure. Results analysis: according to the optimization model of cable-membrane structure form-finding based on particle swarm optimization algorithm, when the initial prestress applied is $10 \mathrm{~N} / \mathrm{cm}$, the form-finding result of the saddle surface cable-membrane structure is optimal. It can be seen from Figure 13 that the surface of the form-finding morphological graph is relatively flat and smooth, and its curvature is relatively small. According to Figure 14(a), it can be obtained that the maximum stress on the film surface is $0.968 \mathrm{kN} / \mathrm{m}$, and the minimum stress is $0.943 \mathrm{kN} / \mathrm{m}$, with a difference of $2.58 \%$. It can be seen that the stress distribution on the film surface is relatively uniform and symmetrical. The maximum stress on the film surface appears at the midpoint of the four sides, and the minimum stress on the film surface appears at the four supports. By comparing Tables 7 and 8 , it can be seen that the saddle surface generated by particle swarm optimization algorithm is more stable than the node coordinate calculated 
TABLE 9: Reaction force of the saddle form-finding support.

\begin{tabular}{lccc}
\hline Node & $\begin{array}{c}\text { Reaction force in the } X \text { direction } \\
(\mathrm{kN})\end{array}$ & $\begin{array}{c}\text { Reaction force in the } Y \text { direction } \\
(\mathrm{kN})\end{array}$ & $\begin{array}{c}\text { Reaction force in the } Z \text { direction } \\
(\mathrm{kN})\end{array}$ \\
\hline The lower left corner & -42.615 & -38.035 & 10.832 \\
The lower right & 42.623 & -38.047 & -10.832 \\
corner & 42.615 & 38.035 & 10.832 \\
The top right corner & -42.623 & 38.047 & -10.832 \\
The upper left corner & & & \\
\hline
\end{tabular}

Note. The node number is numbered upward along the $y$-axis from the center point of the lower edge (including the corner point) in the plane projection of the surface.

by the force density method. Comparing the bearing reaction obtained in Table 9 with that obtained by applying initial prestress, the maximum bearing reaction is the smallest, which proves that this method is correct and the form-finding result is optimal.

Conclusions: according to the abovementioned analysis results, it is feasible to use the particle swarm optimization model of the cable-membrane structure to optimize the form-finding of the saddle surface, which is simple to operate, easy to realize, fast to calculate, and the formfinding result is optimal when the initial prestress applied is $10 \mathrm{~N} / \mathrm{cm}$.

\section{Conclusions}

Form-finding is a key step in the design of membrane structures. Form-finding of cable-membrane structures based on particle swarm optimization algorithm is proposed in this study. The optimization objective, design variables, and constraint conditions are determined in the proposed optimization model. Form-finding of different membrane structures including rotating catenary membrane structure, saddle membrane structure, and rhombic hyperboloid membrane structure is carried out. The optimization results from the proposed PSO are validated since the results compare well with those from the traditional force density method. The initial prestresses of the three membrane structures are obtained while the form-finding result is optimal, respectively. The proposed PSO shows more accurate results in form-finding of cable-membrane structures compared with the traditional force density method. Membrane structures are used widely around the world while the form-finding methods are not so accurate and efficient; intelligent optimization methods such as PSO pave a way for the form-finding of membrane structures in a more accurate and efficient way. Moreover, PSO can be employed in the optimization of membrane structures considering the loading on them such as wind, which will be performed in the future research.

\section{Data Availability}

The data used to support the findings of this study are included within the article.

\section{Conflicts of Interest}

The authors declare that they have no conflicts of interest.

\section{Acknowledgments}

This study was supported by the National Natural Science Foundations of China (no. 51108345), Guangxi Key Laboratory of New Energy and Building Energy Saving Foundation (Gui Keneng 19-J-21-14), Joint Cultivation Program of the National Natural Science Foundations of Guangxi (2019GXNSFAA245037), Guangxi Youth Innovative Talents Research Project (Guike AD19245012), State Key Laboratory of Civil Engineering Disaster Prevention Open Foundations (no. SLDRCE-MB-04), and Scientific and Technology Startup Foundation of Guilin University of Technology (GUTQGJJ2019042 and GUTQDJJ2019041).

\section{References}

[1] H. Zhu, Y. Wu, and W. Zhang, "Theoretical analysis and application of cable-membrane structure," in Proceedings of the 17th National Symposium on Modern Structural Engineering, 2017.

[2] S. Lin, Mechanical Property Analysis of Membrane Structures Under Snow Loads, Zhejiang University, Hangzhou, China, 2017.

[3] S. Yuan, K. Jiang, and Q. Xing, "A new adaptive fem for minimal surfaces form-finding of membrane structures," Engineering Mechanics, vol. 36, no. 1, pp. 15-22, 2019.

[4] A. N. Skvortsov, "Estimation of rotation ambiguity in multivariate curve resolution with charged particle swarm optimization (cPSO-MCR)," Journal of Chemometrics, vol. 28, no. 10, pp. 727-739, 2014.

[5] D. Krause, M. A. Hussein, and T. Becker, "Online monitoring of bioprocesses via multivariate sensor prediction within swarm intelligence decision making," Chemometrics and Intelligent Laboratory Systems, vol. 145, pp. 48-59, 2015.

[6] H. Ghafarzadeh and A. Bouyer, "An efficient hybrid clustering method using an artifificial bee colony algorithm and mantegna Léey distribution," International Journal on Artificial Intelligence Tools, vol. 25, no. 2, 2016.

[7] H. Wang, "Research on parameter adjustment strategy of particle swarm optimization algorithm," Journal of Xinxiang University, vol. 36, no. 12, pp. 27-36, 2019.

[8] N. Di Cesare, D. Chamoret, and M. Domaszewski, "A new hybrid PSO algorithm based on a stochastic Markov chain model," Advances in Engineering Software, vol. 90, pp. 127137, 2015.

[9] Y. Wu, Q. Yang, and S. Shen, "The current status and prospects of analysis theory of membrane structures," Engineering Mechanics, vol. 31, no. 2, pp. 1-14, 2014.

[10] M. Khatibinia and H. Yazdani, "Accelerated multi-gravitational search algorithm for size optimization of truss 
structures," Swarm and Evolutionary Computation, vol. 38, pp. 109-119, 2018.

[11] Q. Zhang and X. Luo, "Inspection and application for existing cable and membrane structures," Construction Technology, vol. 47, no. 6, pp. 41-44, 2018.

[12] M. Dehghani, M. Mashayekhi, and E. Salajegheh, "Topology optimization of double- and triple-layer grids using a hybrid methodology," Engineering Optimization, vol. 48, no. 8, pp. 1333-1349, 2016.

[13] Z. Wang, Deformation Control and Load Analysis of CableMembrane Inflatable Tubes, Harbin Institute of Technology, Harbin, China, 2017.

[14] H. Nenavath, R. K. Jatoth, and S. Das, "A synergy of the sinecosine algorithm and particle swarm optimizer for improved global optimization and object tracking," Swarm and Evolutionary Computation, vol. 43, 2018.

[15] M. Mahmood, S. Mathavan, and M. Rahman, "A parameterfree discrete particle swarm algorithm and its application to multi-objective pavement maintenance schemes," Swarm and Evolutionary Computation, vol. 43, 2018.

[16] Y. Zhang, The Form-Finding and Wind-Induced Response of Tensioned-Membrane Structure Greenhouse, Kunming University of Science \& Technology, Kunming, China, 2010.

[17] J. García-Nieto, E. Lopez-Camacho, M. J. García-Godoy, A. J. Nebro, and J. F. Aldana-Montes, "Multi-objective ligandprotein docking with particle swarm optimizers," Swarm and Evolutionary Computation, vol. 44, 2018.

[18] F. Yang and Y. Gao, "Vibration analysis of membrane structure," Science \& Technology Information, vol. 13, 2013.

[19] R. Zhao, Investigation an Wrinkling of Membranes and Failure of Cable System of Tensioned Cable-Membrane Structures, South China University of Technology, Guangzhou, China, 2011.

[20] H. Su, The Form-Finding Analysis and Calculation of the Tensioned Cable-membrane Structures, Harbin Engineering University, Harbin, China, 2007. 